\title{
Depressive symptoms in adolescence may increase the risk of psychiatric disorders in early adulthood
}

Source of funding:

Medical Society of

Finland; Yrjo Jahnsson

Foundation; Finnish

Cultural Foundation.

For correspondence:

TAalto-Setala,

Department of Mental

Health and Alcohol

Research, National

Public Health Institute,

Finland.

Aalto-Setala T, Marttunen M, Tuulio-Henriksson A et al. Depressive symptoms in adolescence as predictors of early adulthood depressive disorders and maladjustment. Am J Psych 2002 July;159:1235-7.

\section{QUESTION: Do self-reported depressive symptoms in adolescence predict depressive and other psychiatric disorders in early adulthood?}

Design

High school cohort followed for 5 years (1990-1995).

\author{
Setting \\ Two cities in Finland.
}

Relationship between depressive symptoms during adolescence and psychiatric disturbance in early adulthood

\begin{tabular}{llc} 
Early adulthood outcome & $\begin{array}{c}\text { Likelihood of outcome if } \\
\text { depressive symptoms present } \\
\text { in adolescence (odds ratio) }\end{array}$ & $\begin{array}{c}95 \% \text { confidence } \\
\text { interval }\end{array}$ \\
\hline Any DSM-IV axis I disorder & $2.2^{*}$ & 1.1 to 4.3 \\
\hline Axis I comorbidity & $6.2^{*}$ & 2.3 to 16.5 \\
\hline Psychosocial impairment & $3.5^{\star}$ & 1.6 to 7.6 \\
\hline Psychiatric disturbance & $3.4^{*}$ & 2.2 to 5.2 \\
\hline Depressive disorder & $3.2^{*}$ & 1.4 to 7.5 \\
\hline Problem drinking & 1.6 & 1.0 to 2.4 \\
\hline Eating disorder & 2.4 & 0.8 to 7.3 \\
\hline Anxiety disorder & 1.9 & 0.7 to 4.9 \\
\hline Substance use disorder & 0.9 & 0.2 to 3.7 \\
\hline
\end{tabular}

Note: Odds ratio is based on logistic regression adjusting for age, gender and family social class. Statistically significant differences are marked *

\section{COMMENTARY}

Sub-syndromal depression occurs where individuals admit to a number of depressive symptoms, but not enough to meet the criteria for clinical depression. Sub-syndromal depression is associated with a wide range of psychopathology, as well as persistent and recurrent episodes. Aalto-Setala et al suggest that this trend is evident in adolescence and early adulthood. The findings are also compatible with the suggestion that early anxiety and depression may be associated with chronic disorder.

Aalto-Setala et al suggest that adolescents who experience depressive symptoms are more likely to have depression, a range of comorbid conditions and to be psychosocially impaired in early adulthood. An alternative explanation involves attributional style, personality and temperament. Those who tend to "psychologise" may be more likely to state that they "often" get depressed or that they are "continually" depressed, cue words in the current study. This may reflect an individual's temperament and personality: some people may be more likely to develop such symptoms when dealing with the vicissitudes of adolescence or be more likely to use words such as "often" and "continually" when describing their "symptoms." These individuals may also be more likely to suggest a wider range of psychological disorders in later years.

The latter interpretation is supported by the data tabulated above. In early adulthood, those reporting depressive symptoms in adolescence were more likely to meet criteria for almost all disorders, apart from substance abuse and eating disorders. It may therefore be important to broaden the authors' conclusions from "depressive symptoms in adolescence deserve attention as a potential risk for early adulthood mental disorders" to ask "what are the attributional, temperament, personality and related higher-order factors that shape both the early and subsequent expression of depression across adolescence and adulthood?" Rather than reifying constructs such as "sub-syndromal depression" or "sub-clinical depressive symptoms in adolescence," we need to consider broader aetiological factors (especially temperament) in further research and in the clinical application of these important findings.

Professor Gordon Parker, MD PhD DSc Director, Black Dog Institute University of New South Wales Sydney, Australia

\section{Participants}

709 adolescents completed baseline questionnaires during their last three years of high school; mean age 16.8 years. $92 \%$ responded to a follow up questionnaire 5 years later; mean age 21.8 years; $64 \%$ women.

\section{Assessment of risk factors}

Depressive symptoms during adolescence were assessed using 2 items from the 36-item General Health Questionnaire. Family social class was assessed using City of Helsinki social classifications based on father's occupation, or mother's occupation where the father did not live with the adolescent.

\section{Main outcome measures}

Depressive and other psychiatric disorders during early adulthood were assessed using the 36-item General Health Questionnaire. The Schedules for Clinical Assessment in Neuropsychiatry were used to obtain diagnostic interview data from a subgroup of young adults who scored above the cutoff point for "psychiatric disturbance" ( 5 or more symptoms out of 36 ) or whose answers about referrals to mental health services, pathological eating behaviour, alcohol intake or recurrent depressive feelings were cause for concern $(n=245)$. Problem drinking was defined as 2 or more positive answers on the 4-item CAGE questionnaire.

\section{Main results}

Adolescents who reported depressive symptoms while at high school were at greater risk of psychiatric disturbance and problem drinking in young adulthood. Adolescents with depressive symptoms were twice as likely to experience a DSM-IV axis I disorder and 3 times as likely to have major depression or dysthymia in early adulthood compared to those who did not report depressive symptoms during adolescence (table). Depressive symptoms during adolescence did not predict anxiety disorder, eating disorder or substance abuse during early adulthood.

\section{Conclusions}

Self reported depressive symptoms in adolescence predicted depression, psychosocial impairment and problem drinking in early adulthood. 